\title{
Effect of Iron Ore Shape on Gaseous Reduction Rate
}

\author{
Heung Won KANG, Won Sub CHUNG, ${ }^{1)}$ Takeaki MURAYAMA ${ }^{2)}$ and Yoichi ONO ${ }^{3)}$
}

Smelting Reduction Research Team, Research Institute of Industrial Science and Technology (RIST), Pohang 790-600, Korea. 1) Department of Metallurgical Engineering, College of Engineering, Pusan National University, Pusan 609-735, Korea.

2) Department of Materials Science and Engineering, Faculty of Engineering, Kyushu University, Hakozaki, Higashi-ku, Fukuoka, Fukuoka-ken, 812-8581 Japan. tion, Japan.

3) Professor Emeritus, Kyushu University. Now at Kawasaki Steel Corpora-

(Received on March 23, 1998; accepted in final form on July 3, 1998)

\begin{abstract}
In order to clarify the effect of particle shape on the gaseous reduction rate of iron ores, iron oxide pellets of various shapes including the long cylindrical-and the disc shape are reduced with $10 \% \mathrm{CO}-90 \% \mathrm{CO}_{2}$ gas mixture. Experimental results show that the reduction rate of the disc-shaped particle is the fastest among all and the long cylindrical particle follows. The experimental data are analyzed by the unreacted-core model for non-spherical particle proposed by authors in the previous paper. According to the analysis, this model is applicable for the non-spherical iron ore of the cylindrical shape within the ratio range of the height to the cylindrical diameter from 0.5 to 2 while the particles of the long cylindrical- and disc-shaped particle out of this range are problematic applying the model. Based on the review of the experimental-and analysis results, the kinetic behavior concept for the non-spherical particle including the long cylindrical-and disc-shaped particles is discussed.
\end{abstract}

KEY WORDS: shape factor; reduction rate; iron ore; non-spherical particle; non-spherical radius; unreacted-core model.

\section{Introduction}

The kinetic studies on the gaseous reduction of iron ore have been carried out using mainly the spherical pellets. ${ }^{1-3)}$ However, the raw materials used for the real process are not always the spherical shape. Hence, it is necessary to evaluate the shape effect on the gaseous reduction of iron ores to apply their kinetic behavior precisely to the design of the new reactor and the analysis of the existing processes.

The authors have proposed a reduction model for a non-spherical iron oxide particle and reported that the reduction rate increases as the ratio of the surface area to the volume of a non-spherical particle increases. ${ }^{4}$ This model is intended for the shape that the half of the longest side is shorter than the shortest side and the medium side is shorter than the double length of the shortest side when the circumscribed hexahedrons is assumed. In case that the kinetic behavior follows the assumption of this model, the fluxes are oriented to the volumetric center and the instant velocity of the reaction interface at a certain time is proportional to the maximum diffusion length in the proceeding direction. For this reason, the relative thickness of the product layer at a certain time is the same regardless of the layer location and the kinetic equations can be simply modified by the Leva's shape factor. ${ }^{5}$

However, this assumption might not be applied to the long-cylindrical particle of which the side wall area is sufficiently wider than the top- and bottom area of the cylinder or the disc-shaped particle of which the top-and bottom area is much wider than the side wall area. When it is assumed that the reduction proceeds topochemically in the cylindrical particle of which the height is endlessly long, the reaction rate is controlled by the cylindrical radius of the particle. In case of the disc-shaped particle of which the top-and bottom area is endlessly wide, the reaction rate is controlled by the thickness of the particle. These relations are connected by the shape factor proposed by Sohn et al ${ }^{6}{ }^{6}$ Here, a special attention needs to be paid to the fact that there are two different shape factors, Leva's and Sohn's shape factor. However, the similarity or the difference of these two factors has never discussed simultaneously before.

In this study, a long-cylindrical pellet and a discshaped pellet are reduced under the same condition of the previous studies to clarify the shape effect of the particle on the gaseous reduction rate of iron oxide and experimental results are analyzed using the model proposed in the previous study. ${ }^{4}$ Based on this analysis result, the applicability limit of this model is investigated and how the two shape factors affect the kinetics of the gaseous reduction for the iron oxides of various shapes is reviewed. 


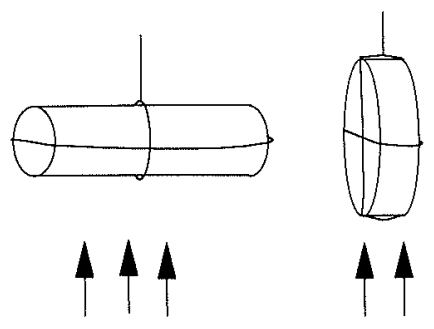

Fig. 1. Flow direction toward sample.

Table 1. Characteristics of samples.

\begin{tabular}{|c|c|c|c|c|}
\hline No & Shape & Dimension $(\mathrm{cm})$ & $\varphi_{L}(-)$ & Remarks \\
\hline \hline P1 & Sphere & $\phi 1.24$ & 1.00 & \\
\hline P2 & Cylinder 1 & $\phi 1.19 \times 0.9$ & 0.86 & $\mathrm{H}_{0} / \mathrm{D}_{0}=0.76$ \\
\hline P3 & Hexahedrons & $1.2 \times 1.2 \times 0.7$ & 0.77 & \\
\hline P4 & Sphere & $\phi 1.56$ & 1.00 & \\
\hline P5 & Cylinder 2 & $\phi 1.66 \times 0.92$ & 0.84 & $H_{0} / D_{0}=0.56$ \\
\hline P6 & Long cylinder & $\phi 0.83 \times 1.9$ & 0.80 & $H_{0} / D_{0}=2.3$ \\
\hline P7 & Disc & $\phi 1.55 \times 0.53$ & 0.76 & $H_{0} / D_{0}=0.34$ \\
\hline
\end{tabular}

\section{Experimental}

\subsection{Experimental Method and Condition}

Experimental apparatus is omitted in this paper because it was described in the previous paper. ${ }^{7}$ An iron oxide pellet of particular shape fabricated using the reagent grade of hematite powder is hung in the reaction tube (inner diameter: $3.5 \mathrm{~cm}$ ) as shown in Fig. 1 and reduced with $10 \% \mathrm{CO}-90 \% \mathrm{CO}_{2}$ gas mixture at the temperature of 700,800 and $900^{\circ} \mathrm{C}$. The flow rate is $4 \mathrm{~N} / \mathrm{min}$. The reduction step from hematite to magnetite is adopted as done in the previous studies. ${ }^{4.7)} \mathrm{It}$ is because in this step the applicable condition of the unreacted-core model can be easily obtained due to denser product layer and larger Thiele's modulus $\phi^{8)}$ than the other steps and because swelling and cracking during the first step can affect the kinetics of the latter steps. The weight loss by reduction is measured by a thermal balance and recorded by a pen recorder. The samples are fabricated based on the classification method of the iron ore shape presented by Shin et $a l .{ }^{91}$ as done in the previous study. ${ }^{4,7)}$ They have classified the iron ore shape as the granular, columnar and plate shape and reported that more than $80 \%$ of iron ores in the raw material yard of a steelmaking works can be classified into the granular shape. The granular shape is defined as the shape that the half of the longest side is shorter than the shortest side and the medium side is shorter than the double length of the shortest side when the circumscribed hexahedrons are assumed. The columnar shape is the shape that the longest side is longer than the double length of the medium side. The plate shape is the shape that the longest side is shorter than the double length of the medium side but longer than the double length of the shortest side. Table 1 shows the characteristics of the samples.

According to the above classification method, samples P1, P2, P3, P4 and P5 can be classified into the granular shape, sample P6 into the columnar shape and sample P7 into the plate shape. $\phi_{\mathbf{L}}$ in Table 1 is Leva's shape factor $^{5)}$ and it is defined as the ratio of the surface area
Table 2. Crack during reduction.

\begin{tabular}{|c|c|c|c|c|c|}
\hline \multicolumn{2}{|c|}{ Sample $(\phi L)$} & $700^{\circ} \mathrm{C}$ & $800^{\circ} \mathrm{C}$ & $900^{\circ} \mathrm{C}$ & Remarks \\
\hline \multirow{2}{*}{$P 1(1)$} & $F$ & $G$ & $G$ & $G$ & \\
\hline & $\mathrm{L}$ & $G$ & G & $G$ & \\
\hline \multirow{2}{*}{$P 2(0.86)$} & $F$ & $w$ & $\bar{G}$ & $\mathrm{G}$ & \\
\hline & $\mathrm{L}$ & $w$ & $G$ & $G$ & \\
\hline \multirow[b]{2}{*}{$P 3(0.77)$} & $F$ & $w$ & $G$ & $G$ & \\
\hline & L & $w$ & $G$ & $\mathrm{G}, \mathrm{W}$ & \\
\hline \multirow{2}{*}{ P4(1) } & $F$ & $G$ & $\bar{G}$ & $G$ & \\
\hline & $L$ & $G$ & $G$ & $G$ & \\
\hline \multirow[b]{2}{*}{$P 5(0.84)$} & $F$ & $w$ & $G$ & $G$ & \\
\hline & $\mathrm{L}$ & $w$ & $G$ & $G, W$ & \\
\hline \multirow[b]{2}{*}{$P G(0.80)$} & $F$ & $w$ & $\mathrm{G}$ & $G$ & \multirow{2}{*}{$\begin{array}{l}\text { Long } \\
\text { cylinger }\end{array}$} \\
\hline & $\mathrm{L}$ & $w$ & $\mathrm{G}$ & $w$ & \\
\hline \multirow[b]{2}{*}{$P 7(0.76)$} & $F$ & $w$ & $G$ & $G$ & \multirow[b]{2}{*}{ Disc } \\
\hline & $\mathrm{L}$ & $w$ & G & $w$ & \\
\hline
\end{tabular}

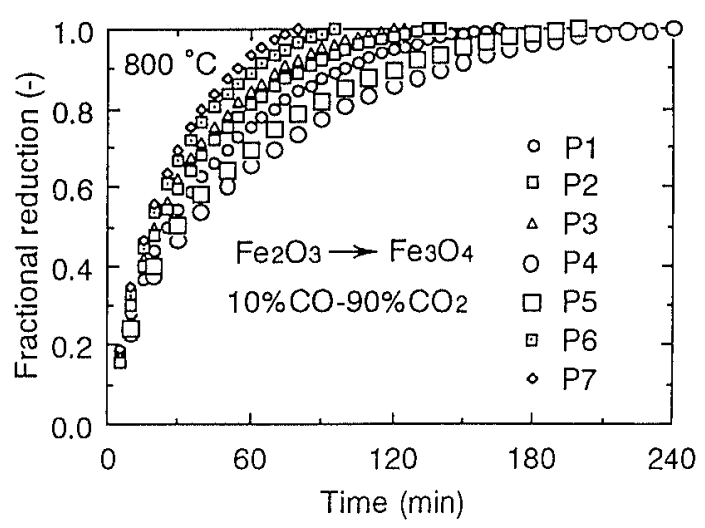

Fig. 2. Effect of particle shape on reduction rate.

of the sphere of the same volume as a non-spherical particle to that of the particle.

\subsection{Experimental Results}

Cracks have occurred every experimental run carried out at $700^{\circ} \mathrm{C}$ except the spherical samples $\mathrm{P} 1$ and $\mathrm{P} 4$. Cracks have also occurred in samples $\mathrm{P} 6$ and $\mathrm{P} 7$ from the middle stage of the reduction at $900^{\circ} \mathrm{C}$. However, cracking has less occurred for all the shapes of the samples at $800^{\circ} \mathrm{C}$. In Table 2, the result of cracking during reduction is summarized. Table 2 shows clearly that cracking during the reduction of the iron oxide is affected by the iron oxide shape and the reduction temperature.

Figure 2 shows the reduction curves for all samples of various shapes where samples P1, P2, P3, P6 and P7 weigh $4 \mathrm{~g}$ and samples $\mathrm{P} 4$ and $\mathrm{P} 5$ are $8 \mathrm{~g}$. Among the above samples, the reduction rate of sample $\mathrm{P} 7$ which is disc-shaped is the fastest. That of sample P6 which is long-cylindrical follows.

In the previous study, ${ }^{4}$ it has been reported that the reduction rate becomes faster as the value of $\phi_{\mathrm{L}}$ becomes smaller. The shape factor $\phi_{\mathrm{L}}$ of sample P6 is 0.80 and this value is larger than that of sample $\mathrm{P} 3$ of which $\phi_{\mathbf{L}}$ is 0.77 . Nevertheless, the rate of sample $\mathrm{P} 6$ is faster than that of sample P3. Sample P7 has 0.76 in $\phi_{\mathrm{L}}$ but the reduction rate is much faster than that of sample P3. By this reason, it can be induced that the reduction rate 
cannot be predicted only by the non-spherical radius, that is, the iron oxide size and Leva's shape factor. This implies that the maximum diffusion length of samples $\mathrm{P} 6$ and $\mathrm{P} 7$ is not the non-spherical radius. In this study, the reduction data of these two samples are analyzed by the gas-solid reaction model for non-spherical iron oxide proposed by the authors in the previous paper ${ }^{4}$ to check the limitation of the model (hereafter it will be called as the non-spherical model).

\section{Non-spherical Model ${ }^{4)}$}

\subsection{Overall Rate Equation}

Now, let us consider a class of gas-solid reactions represented by Eq. (1). If it is assumed that the reaction in a non-spherical particle proceeds topochemically in the manner that the fluxes are oriented to the volumetric center and the moving velocity of the reaction interface at a certain time is proportional to the maximum diffusion length at the proceeding direction, this reaction proceeds through the five steps occurring successively.

$$
\mathrm{R}(\text { solid })+\mathrm{A}(\text { gas })=\mathrm{P}(\text { solid })+\mathrm{B}(\text { gas })
$$

\subsubsection{Gas-Film Diffusion}

If the gas composition in the bulk gas phase outside the film is assumed to be uniform, the rates of transfer of reducing gas "A" and product gas "B" through the gas-film are respectively given by

$$
\begin{aligned}
& -\dot{n}_{\mathrm{g}, \mathrm{A}}=k_{\mathrm{gA}} \cdot \frac{4 \pi r_{\mathrm{o}}^{2}}{\phi_{\mathrm{L}}} \cdot\left(C_{\mathrm{A}}^{\mathrm{b}}-C_{\mathrm{A}}^{\mathrm{o}}\right) \\
& +\dot{n}_{\mathrm{g}, \mathrm{B}}=k_{\mathrm{gB}} \cdot \frac{4 \pi r_{\mathrm{o}}^{2}}{\phi_{\mathrm{L}}} \cdot\left(C_{\mathrm{B}}^{\mathrm{o}}-C_{\mathrm{B}}^{\mathrm{b}}\right)
\end{aligned}
$$

\subsubsection{Intraparticle Diffusion}

The molar flow of reactant or product gas across any non-spherical surface in the product layer is given by

$$
\dot{n}_{j}=-\left(4 \pi r^{2} / \phi_{\mathrm{L}}\right) \cdot D_{\mathrm{e} j} \cdot \frac{\partial C_{j}}{\phi_{\mathrm{L}} \partial r} \quad(j=\mathrm{A} \text { or } \mathrm{B})
$$

If Eq. (4) is integrated with respect to $r$ between the reaction interface at $r_{\mathrm{i}}$ and exterior surface at $r_{0}$, the rates of transfer of reactant and product gases through the product layer are respectively given by

$$
\begin{aligned}
& -\dot{n}_{\mathrm{d}, \mathrm{A}}=D_{\mathrm{eA}} \cdot \frac{4 \pi r_{\mathrm{o}} r_{\mathrm{i}}}{\phi_{\mathrm{L}}^{2}\left(r_{\mathrm{o}}-r_{\mathrm{i}}\right)} \cdot\left(C_{\mathrm{A}}^{\circ}-C_{\mathrm{A}}^{\mathrm{i}}\right) \\
& +\dot{n}_{\mathrm{d}, \mathrm{B}}=D_{\mathrm{eB}} \cdot \frac{4 \pi r_{0} r_{\mathrm{i}}}{\phi_{\mathrm{L}}^{2}\left(r_{\mathrm{o}}-r_{\mathrm{i}}\right)} \cdot\left(C_{\mathrm{B}}^{\mathrm{i}}-C_{\mathrm{B}}^{\circ}\right)
\end{aligned}
$$

\subsubsection{Interface Chemical Reaction}

If the interface reaction is assumed to be first-order and reversible, the rate of the reaction is given by

$$
\dot{n}_{\mathrm{c}}=k_{\mathrm{c}} \cdot \frac{4 \pi r_{\mathrm{i}}^{2}}{\phi_{\mathrm{L}}} \cdot\left(C_{\mathrm{A}}^{\mathrm{i}}-C_{\mathrm{B}}^{\mathrm{i}} / K\right)
$$

\subsubsection{Overall Rate Equation}

For every gram atom of oxygen removed from the oxide, one mole of reducing gas is consumed and one mole of product gas is formed, so that at steady state:

$$
\dot{n}=-\dot{n}_{\mathrm{g}, \mathrm{A}}=-\dot{n}_{\mathrm{d}, \mathrm{A}}=\dot{n}_{\mathrm{c}}=\dot{n}_{\mathrm{d}, \mathrm{B}}=\dot{n}_{\mathrm{g}, \mathrm{B}}
$$

Upon substitution of Eqs. (3), (4), (5), (6) and (7) for Eq. (8), the interface and surface concentration terms are canceled out, yielding the following overall rate equation:

$$
\dot{n}=\frac{\phi_{\mathrm{L}}}{\frac{C_{\mathrm{A}}^{\mathrm{b}}-C_{\mathrm{A}}^{\mathrm{e}}}{k_{\mathrm{g}} \cdot 4 \pi r_{\mathrm{o}}^{2}}+\frac{\phi_{\mathrm{L}}^{2}\left(r_{\mathrm{o}}-r_{\mathrm{i}}\right)}{D_{\mathrm{e}} \cdot 4 \pi r_{\mathrm{o}} r_{\mathrm{i}}}+\frac{\phi_{\mathrm{L}}}{k_{\mathrm{c}} \cdot 4 \pi r_{\mathrm{i}}^{2}(1+1 / K)}}
$$

where

$$
\begin{aligned}
& \frac{1}{k_{\mathrm{g}}}=\left(\frac{1}{k_{\mathrm{gA}}}+\frac{1}{k_{\mathrm{gB}} \cdot K}\right) \cdot\left(\frac{K}{1+K}\right) \cdots \\
& \frac{1}{D_{\mathrm{e}}}=\left(\frac{1}{D_{\mathrm{eA}}}+\frac{1}{D_{\mathrm{eB}} \cdot K}\right) \cdot\left(\frac{K}{1+K}\right)
\end{aligned}
$$

$\dot{n}_{\mathrm{c}}$ is related to $-\phi_{\mathrm{L}} d r_{\mathrm{i}} / d t$ by

$$
\dot{n}_{\mathrm{c}}=-\frac{d}{d t}\left(V_{\mathrm{i}} \cdot d_{\mathrm{R}}\right)=-\frac{4 \pi r_{\mathrm{i}}^{2} \cdot d_{\mathrm{R}}}{\phi_{\mathrm{L}}} \cdot \frac{\phi_{\mathrm{L}} d r_{\mathrm{i}}}{d t}
$$

Using the relative thickness of the product layer $f$ defined by

$$
f=1-(1-F)^{1 / 3}
$$

relation between $f$ and reaction time $t$ is given by

$$
\begin{aligned}
& \frac{C_{\mathrm{A}}^{\mathrm{b}}-C_{\mathrm{A}}^{\mathrm{e}}}{\phi_{\mathrm{L}} r_{\mathrm{o}} d_{\mathrm{R}}} \cdot \frac{t}{f}-\frac{3-3 f+f^{2}}{3 k_{\mathrm{g}}} \\
& \quad=\frac{\phi_{\mathrm{L}} r_{\mathrm{o}}}{6 D_{\mathrm{e}}} \cdot\left(3 f-2 f^{2}\right)+\frac{1}{k_{\mathrm{c}}(1+1 / K)}
\end{aligned}
$$

Here, let

$$
\begin{array}{r}
X=3 f-2 f^{2} \ldots \ldots \ldots \ldots \\
Y=\frac{C_{\mathrm{A}}^{\mathrm{b}}-C_{\mathrm{A}}^{\mathrm{e}}}{\phi_{\mathrm{L}} r_{\mathrm{o}} d_{\mathrm{R}}} \cdot \frac{t}{f}-\frac{3-3 f+f^{2}}{3 k_{\mathrm{g}}}
\end{array}
$$

Equation (14) is simplified as $Y=\alpha X+\beta$. Therefore, if $Y$ calculated from the experimental data is plotted against $X$, a straight line would be obtained in case that the assumption of this model is acceptable. The intraparticle diffusivity through the product layer $D_{\mathrm{e}}$ is determined from the slope of this line and the interface chemical reaction rate constant $K_{\mathrm{c}}$ from the intercept of the line with ordinate axis as Yagi et al.'s analytical method by the mixed control plot. ${ }^{10)}$

\subsubsection{Determination of Gas-Film Mass Transfer Co- efficient for Non-spherical Particle}

It is reported that Gokarn et al. ${ }^{11)}$ used $r_{\mathrm{s}}$, the radius of the sphere of the same surface area as a non-spherical particle when he evaluated the mass transfer coefficient $k_{\mathrm{g}}$ based on the concept of the Satterfield et al. ${ }^{12)}$ in the oxidation experiment of $\mathrm{ZnS}$ pellets of various shapes. In this study, $k_{\mathrm{g}}$ is estimated using Gokarn et al.'s method as done in the previous study, that is, using the following correlation of modified Ranz and Marshall's equation ${ }^{13)}$ :

$$
\frac{k_{\mathrm{g}}\left(2 r_{\mathrm{s}}\right)}{D_{\mathrm{AB}}}=2+0.6\left(\frac{2 \rho r_{\mathrm{o}} u}{\mu}\right)^{1 / 2}\left(\frac{\mu}{\rho D_{\mathrm{AB}}}\right)^{1 / 3}
$$




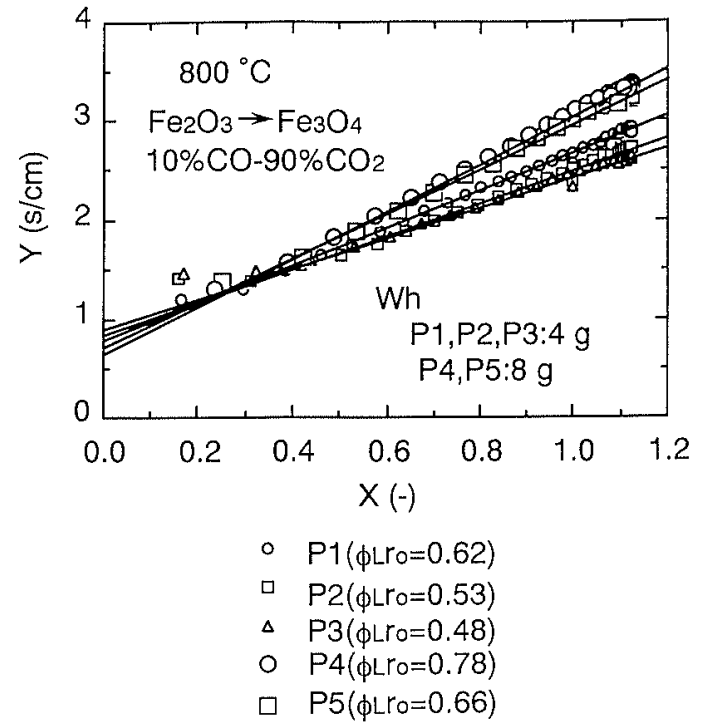

Fig. 3. Mixed-control plots calculated by the non-spherical model for samples P1, P2, P3, P4 and P5.

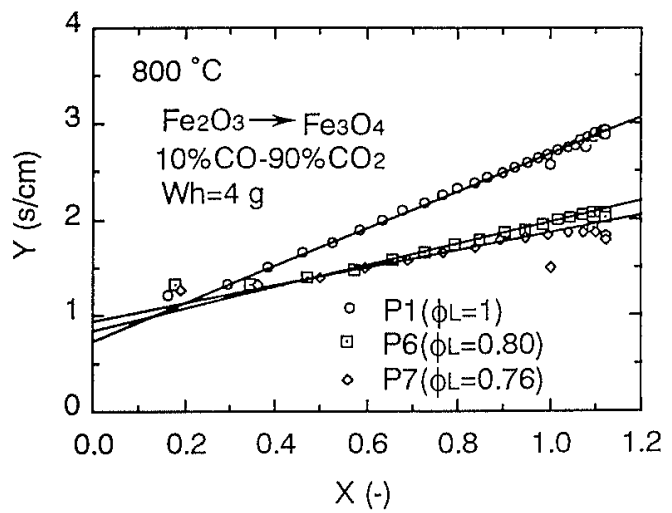

Fig. 4. Mixed-control plots calculated by the non-spherical model for the long-cylindrical particle P6 and the disc-shaped particle P7.

$k_{\mathrm{g}}$ is reestimated as $k_{\mathrm{f}}$ using the following equation considering the dilution effect. ${ }^{14)}$

$$
k_{\mathrm{f}}=1 /\left(1 / k_{\mathrm{g}}+4 \pi r_{\mathrm{s}}^{2} / V_{\mathrm{T}}\right)
$$

The various constants are calculated in the same equations used in the previous paper. ${ }^{15)}$

\section{Analysis Results and Discussion}

\subsection{Analysis Results}

The mixed-control plots calculated by the nonspherical model ${ }^{4)}$ are shown in Figs. 3 and 4 . Figure 3 shows the mixed-control plots for the samples P1, P2, P3, P4 and P5 classified into the granular shapes according to Shin et al.'s classification method. ${ }^{93}$ Figure 4 shows the mixed-control plots for samples P6 and P7 classified into the columnar- and plate shape respectively, where that of sample Pl are loaded for comparison. As can be seen in Fig. 3, samples of the granular shapes are showing an excellent straightness in the mixed-control plots. This results are well reflected in the simulation of the reduction curves in Fig. 5. The calculated reduction curves are in good agreement with the observed ones. However, the slopes of the mixed-control plots for samples
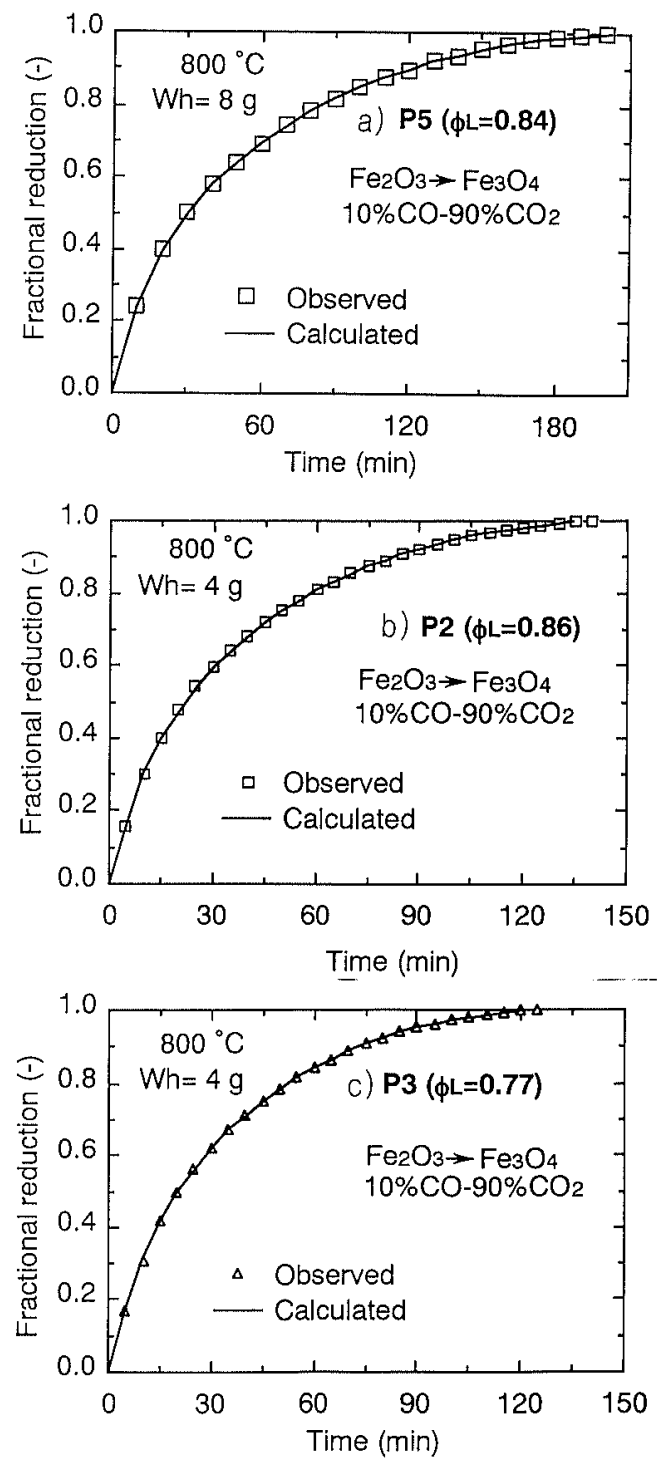

Fig. 5. Comparison of the reduction curves for samples $P 2$, P3 and P5 calculated by the non-spherical model with the observed one

P6 and P7 are much smaller than that of sample P1. In addition, those plots are slightly deviated from the linearity at the final stage of the reduction. Especially, this trend is more remarkable for sample P7. As the result, the calculated reduction curves are deviated a little from the observed ones as can be seen in Fig. 6. This is because the reduction rates of samples P6 and P7 are faster than values calculated by the non-spherical mod$\mathrm{el}^{4)}$ at the final stage of reduction.

The authors have reported in the previous papers ${ }^{4,7)}$ that the effective diffusivity through the product layer $D_{\mathrm{e}}$ does not depend on the radius or non-spherical radius of a particle but the interface chemical reaction rate constant $k_{\mathrm{c}}$ does.

In Fig. 7, the relation between $k_{\mathrm{c}}$ and the non-spherical radius is presented. The values of $k_{\mathrm{c}}$ for samples $\mathrm{P} 6$ and P7 seem to be the same trend as those of others.

However, the values of $D_{\mathrm{e}}$ of samples P6 and P7 are larger than those of the others by 0.025 and $0.035 \mathrm{~cm}^{2} / \mathrm{s}$ respectively as can be seen in Fig. 8 . If the kinetic behavior of samples P6 and P7 follows the concept of the 

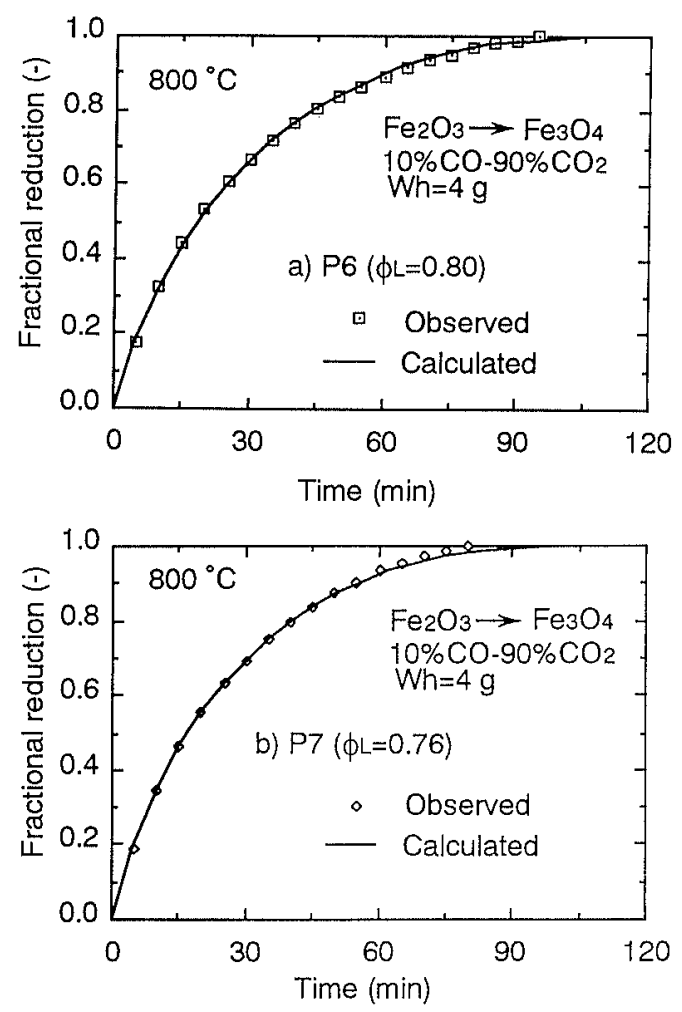

Fig. 6. Comparison of the reduction curves for samples P6 and P7 calculated by the non-spherical model with the observed one.

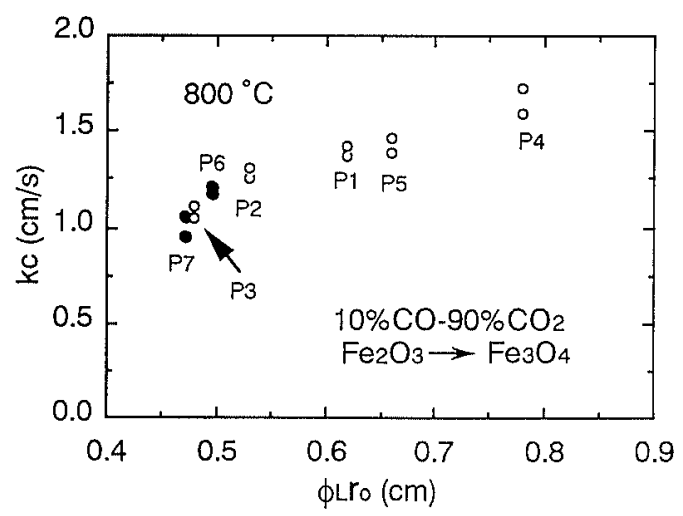

Fig. 7. Relation between the interface chemical reaction rate constant $k_{\mathrm{c}}$ calculated by the non-spherical model and the non-spherical radius.

non-spherical model, ${ }^{4)}$ the obtained values of $D_{\mathrm{e}}$ should be the same as the other samples, because the porosity and labyrinth factor of samples P6 and P7 are the same as those of other samples since these two samples are fabricated in the same manner as the other samples. This is because the maximum diffusion length for samples P6 and $\mathrm{P} 7$ might not be given by the non-spherical radius.

In order to confirm this, the section of the $90 \%$ converted sample P7 has been observed in Fig. 9. As can be seen in Fig. 9, the absolute thickness is different with direction. The thickness of the radius direction is larger than that of the thickness direction while the relative thickness shows the opposite result. The reaction interface of the thickness direction has proceeded more than $50 \%$ to the center while that of the radius has been staying around $30 \%$. From this observation, it

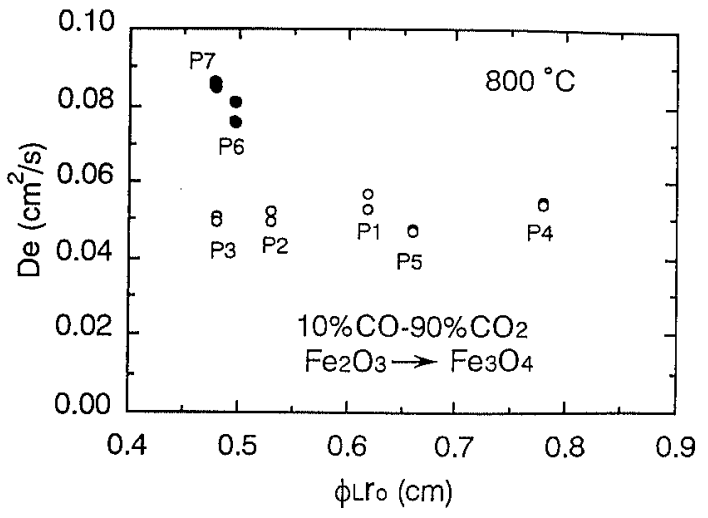

Fig. 8. Relation between the effective diffusivity $D_{\mathrm{c}}$ calculated by the non-spherical model and the non-spherical radius.

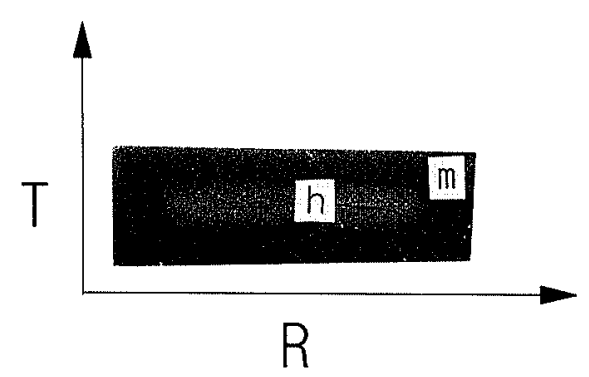

h: Hemat ite
$\mathrm{m}:$ Magnet i te

Fig. 9. Cross-sectional view sample of the fractional reduction $90 \%$ for $\mathrm{P} 7$ reduced at $800^{\circ} \mathrm{C}$.

can be assumed that the interface of the thickness direction has arrived at the center before that of the radius direction does in the iron oxide which is plateshaped like sample P7.

The above result says that the calculated reaction curves corresponding to the observed ones cannot be the only factor for the judgement of the applicability. In order to make the right judgement, it is necessary to refer to the linearity of the mixed-control plot as well as the obtained value of $D_{\mathrm{e}}$.

\subsection{Reaction Rate and Sohn et al.'s Shape Factor}

It seems that the fact that the obtained values of $D_{\mathrm{e}}$ are different from those of other samples is due to the fact that the different Sohn et al.'s shape factor ${ }^{6)}$ affects the kinetic behavior for samples $\mathbf{P} 6$ and $\mathbf{P} 7$ than that of other samples. According to Sohn et al., the maximum diffusion length $L_{\mathrm{p}}$ can be given by

$$
L_{\mathrm{p}}=\frac{Z \cdot V_{\mathrm{p}}}{S_{\mathrm{p}}}
$$

where $V_{\mathrm{p}}$ is the volume of a solid particle, $S_{\mathrm{p}}$ the surface area and $Z$ is Sohn et al.'s shape factor which can be given as 1,2 and 3 respectively to the disc, long cylinder and sphere.

Therefore, it can be said that 3 is applied to samples P1 to P5 which are classified into the granular shape. On the contrary, however, 2 is applied to sample P6 which is columnar and 1 is applied to sample P7 which is 


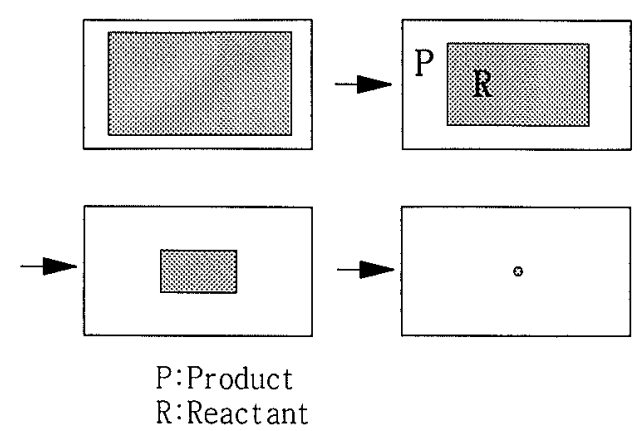

Fig. 10. Two-dimensional conceptual diagram for the kinetic behavior of the granular particle.

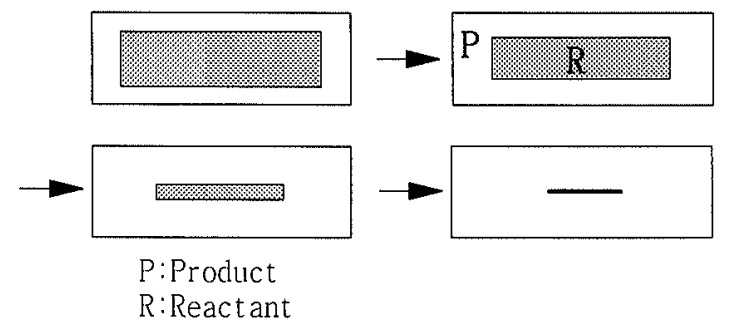

Fig. 11. Two-dimensional conceptual diagram for the kinetic behavior of the columnar- and the plate-shaped particle.

plate-shaped. In such cases, the maximum diffusion length of sample P6 is the cylindrical radius and that of sample $\mathrm{P} 7$ is the half of the thickness of the disc.

This is the reason why the rate of samples P6 is faster than those of sample P3 of which $\phi_{\mathrm{L}}$ is smaller. Further, it is the reason why much larger values of $D_{\mathrm{e}}$ are obtained for samples P6 and P7 than those of the other samples which are classified into the granular shape when they are analyzed by the non-spherical model. ${ }^{4)}$

The structural model proposed by Sohn et al., ${ }^{6)}$ however, considers the mass transportation from the particular direction and is intended for the ideal shape such as the sphere, the long-cylinder of which the height is endlessly long and the disc of which the thickness is nothing comparing to the diameter. Therefore, this model cannot be applied to the iron oxide which is shaped as samples P6 and P7. In order to analyze the kinetics precisely, it is necessary to develop a model which can consider the mass transportation from every direction.

\subsection{Kinetic Concept of Non-spherical Iron Oxide}

In order to clarify the kinetic concept of the granular particle as well as columnar- and plate-shaped particles, the kinetic behavior concepts for the non-spherical particles including the columnar- and plate-shaped particles are schematized.

Figure $\mathbf{1 0}$ is the 2-dimensional conceptual diagram for the kinetic behavior of the granular particle. The unreacted core maintains the original shape of the particle as the reaction proceeds.

Figure 11 is the 2-dimensional conceptual diagram for the kinetic behavior of the columnar- and plate-shaped particle. As can be seen in Fig. 11, the unreacted core becomes thinner and thinner as the reaction proceeds and finally it becomes a segment. This concept might seem to be the same as that of the cylinder model proposed by Gokarn et al. However, the former assumes that the thickness of the product layer is different with the location, while the latter assumes that the thickness of the product layer is the same regardless of the layer location.

\section{Conclusions}

In order to investigate the effect of the iron ore shape on the gaseous reduction rate, the iron oxide pellets of various shapes including the columnar- and plate shape are reduced from hematite to magnetite with $10 \% \mathrm{CO}$ $90 \% \mathrm{CO}_{2}$ gas mixture and the experimental data are analyzed by the non-spherical model. The conclusions are as follows:

(1) The reduction rate of the plate shape is the fastest among the sample of the same weight and that of the long-cylindrical shape follows.

(2) Among the granular particles, the reduction rate becomes faster as the non-spherical radius becomes smaller.

(3) Sample P6 of which the height is longer than the double length of the cylindrical diameter does not follow the concept of the non-spherical model.

(4) Sample P7 of which thickness is shorter than the cylindrical radius does not follow the concept of the non-spherical model.

(5) The non-spherical model can be applicable at the ratio range of the height to the cylindrical diameter from 0.5 to 2 .

(6) The rate of sample P6 is controlled by the cylindrical radius and that of sample $\mathrm{P} 7$ by the half of the thickness of the disc.

\section{Nomenclature}

$C_{\mathrm{A}}^{\mathrm{b}}$ : Bulk concentration of gas $\mathrm{A}\left(\mathrm{mol} / \mathrm{cm}^{3}\right)$

$C_{\mathrm{A}}^{\mathrm{e}}:$ Equilibrium concentration of gas $\mathrm{A}\left(\mathrm{mol} / \mathrm{cm}^{3}\right)$

$C_{\mathrm{A}}^{\mathrm{i}}$ : Concentration of gas $\mathrm{A}$ at the reaction interface $\left(\mathrm{mol} / \mathrm{cm}^{3}\right)$

$C_{A}^{0}$ : Concentration of gas $A$ at the exterior surface of the pellet $\left(\mathrm{mol} / \mathrm{cm}^{3}\right)$

$D_{\mathrm{c}}$ : Effective diffusivity through the product layer included in the unreacted-core model $\left(\mathrm{cm}^{2} / \mathrm{s}\right)$

$D_{\mathrm{AB}}$ : Molecular diffusivity of binary gas mixture A-B $\left(\mathrm{cm}^{2} / \mathrm{s}\right)$

$D_{\mathrm{o}}$ : Cylindrical diameter of a cylindrical particle (cm)

$d_{\mathrm{R}}$ : Concentration of reducible oxygen in the oxide (g-atom $/ \mathrm{cm}^{3}$ )

$F:$ Fractional reduction $(-)$

$f$ : Relative thickness of product layer $(-)$

$H_{\mathrm{o}}$ : Height (thickness) of a cylindrical particle $(\mathrm{cm})$

$k_{\mathrm{c}}$ : Interface chemical reaction rate constant included in the unreacted-core model $(\mathrm{cm} / \mathrm{s})$

$K: \quad$ Equilibrium constant (-)

$k_{\mathrm{g}}$ : Mass transfer coefficient through gas-film $(\mathrm{cm} / \mathrm{s})$

$k_{\mathrm{f}}$ : Mass transfer coefficient through gas-film considering the dilution effect $(\mathrm{cm} / \mathrm{s})$

$r_{\mathrm{i}}$ : The radius of sphere having the same volume as 
the non-spherical unreacted core $(\mathrm{cm})$

$r_{0}$ : The radius of sphere having the same volume as the non-spherical particle $(\mathrm{cm})$

$r_{\mathrm{s}}$ : The radius of the sphere of the same surface area as a non-spherical particle $(\mathrm{cm})$

$t$ : Reaction time (min)

$u$ : Superficial velocity of gas stream $\left(\mathrm{g} / \mathrm{cm}^{2} \cdot \mathrm{s}\right)$

$Z$ : Sohn's shape factor $(-)$

Greek letters

$\Phi: \quad$ Thiele's modulus (-)

$\phi_{\mathrm{L}}$ : Leva's shape factor $(-)$

$\mu$ : Gas viscosity in gas-film $(\mathrm{g} / \mathrm{cm} \cdot \mathrm{s})$

$\rho:$ Gas density in gas-film $\left(\mathrm{g} / \mathrm{cm}^{3}\right)$

\section{REFERENCES}

1) R. H. Spitzer, F. S. Manning and W. O. Philbrook: Trans. Metall. Soc., AIME, 236 (1966), 1715.

2) M. Ishida and C. Y. Wen: AlchE J., 14 (1968), 311.
3) Y. Hara: Tetsu-to-Hagané, 57 (1971), 1441.

4) H. W. Kang, W. S. Chung, T. Murayama and Y. Ono: ISIJ Int., 38 (1998), 324

5) M. Leva: Chem. Eng. Prog., 48 (1947), 549.

6) H. Y. Sohn and J. Szekely: Chem. Eng. Sci, 27 (1972), 763

7) H. W. Kang, W. S. Chung and T. Murayama: ISIJ Int., 38 (1998), 109.

8) E. W. Thiele: Ind. Eng. Chem., 31 (1939), 916.

9) H. K. Shin, D. W. Lee, I. K. Suh and D. Y. Lee: The nature and characteristics of iron ore, RIST, Pohang, (1994), 78.

10) T. Yagi and Y. Ono: Trans. Iron Steel Inst. Jpn., 8 (1968), 377.

11) A. N. Gokarn and L. K. Doraiswamy: Chem. Eng. Sci, 3 (1973), 401 .

12) C. N. Satterfield: Mass Transfer in Heterogeneous Catalysis, MIT Press, Cambridge, Mass., (1970).

13) W. E. Ranz and W. R. Marshall: Chem. Eng. Prog., 48 (1952), 141 .

14) H. W. St. Clair: Trans. Metall. Soc. AIME, 233 (1965), 1145.

15) H. W. Kang, U. C. Chung, W. S. Chung, I. O. Lee and Y. Ono: J. Korean Inst. Met. Mater., 35 (1997), 326. 